\title{
Disrupting MRSA 'persisters'
}

synthetic
retinoids ...
exhibit a high
selectivity
towards MRSA
persisters

The treatment of methicillin-resistant Staphylococcus aureus (MRSA) infections is limited by the emergence of metabolically inactive, dormant 'persisters' that are resistant to conventional antibiotics, making the pursuit of novel antibacterial drugs an imperative. Now, a study published in Nature has identified a potential new class of antibiotics - synthetic retinoids - that can kill both growing and persister MRSA cells by disrupting the bacterial membrane.

Using a Caenorhabditis elegansMRSA infection assay to screen $\sim 82,000$ synthetic molecules, the investigators identified 185 synthetic compounds that rescued nematodes from MRSA-induced death. Two

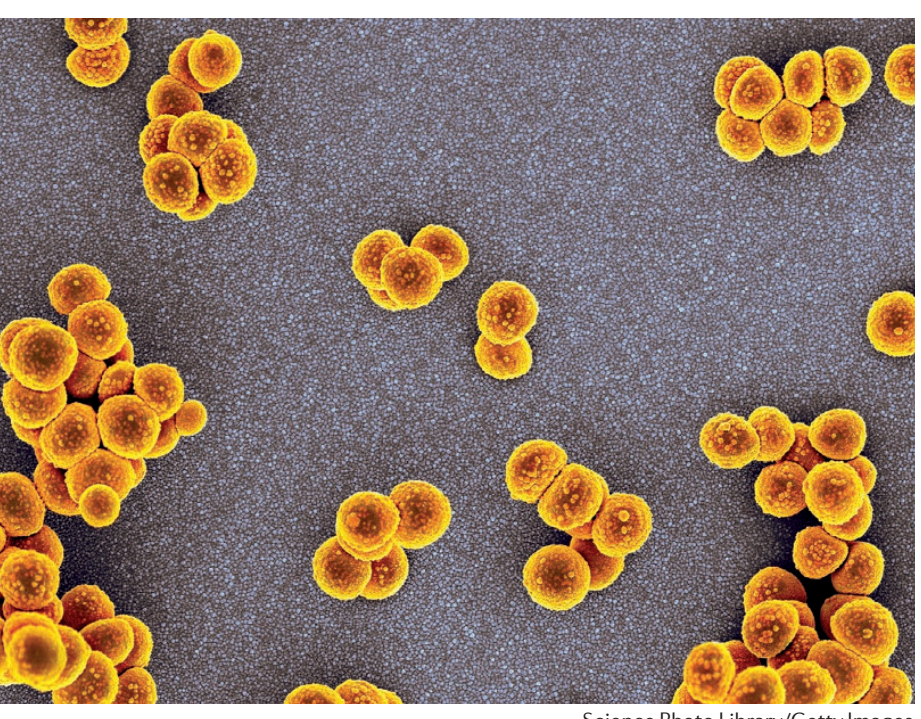

Science Photo Library/Getty Images synthetic retinoids - CD437 and CD1530 - were selected owing to their structural similarity and exhibited potent bactericidal activity against MRSA strain MW2. Strikingly, there was a very low probability of resistance selection upon serial passage of the MW2 strain in the presence of sublethal retinoid doses. Importantly, however, the few resultant retinoid-resistant strains harboured mutations in membrane-related genes, offering a clue to the bactericidal mechanism.

Using all-atom molecular dynamics simulations to model retinoid-membrane interactions, Kim and colleagues then found that the polar branch groups of retinoids bind to the hydrophilic heads of membrane lipids, facilitating bilayer penetration and, ultimately, membrane perturbation. Indeed, the membrane permeabilization activity of retinoids correlated with antibiotic activity. CD437 and CD1530 induced rapid membrane permeabilization and death in MRSA persister cells and biofilms, but were relatively non-toxic in human cell lines. Interestingly, retinoids exhibited synergism with the aminoglycoside antibiotic gentamicin - which is usually ineffective against persisters - in growing and persister cells, which the authors attributed to increased passive diffusion owing to retinoid-induced membrane permeabilization.
To investigate the effects of branch group modification on antimicrobial activity and cytotoxicity, the authors synthesized 16 structural analogues of CD437. Structure-function experiments illustrated that, compared with CD437, analogue 2 - which has a less polar group — retained similar anti-persister activity and was less cytotoxic. In a mouse deep-seated MRSA infection model, treatment with analogue 2 alone - which had a favourable pharmacokinetic and toxicity profile - led to a $\sim 4$-fold decrease in bacterial burden. Furthermore, analogue 2 in combination with gentamicin resulted in a 23 -fold reduction in bacterial burden.

Overall, the study has identified a specific chemotype of membraneactive synthetic retinoids that exhibit a high selectivity towards MRSA persisters, warranting the further investigation of retinoid analogues as antibacterial drugs. Although the potential cytotoxicity of such membrane-active agents is an important but challenging obstacle, optimization of the chemical backbone and branch groups of the $>4,000$ known retinoids could offer a promising solution to minimize toxicity.

Conor A. Bradley

ORIGINAL ARTICLE Kim, W. et al. A new class of synthetic retinoid antibiotics effective against bacterial persisters. Nature 556, 103-107 (2018) 